\title{
Photoreduction and Thermal Properties of Graphene-Based Flexible Films
}

\author{
Gautam Naik, Sridhar Krishnaswamy \\ Northwestern University, Department of Mechanical Engineering, Evanston, Illinois, USA \\ Email: gautamnaik2014@u.northwestern.edu
}

How to cite this paper: Naik, G. and Krishnaswamy, S. (2017) Photoreduction and Thermal Properties of Graphene-Based Flexible Films. Graphene, 6, 27-40. https://doi.org/10.4236/graphene.2017.62003

Received: March 13, 2017

Accepted: April 22, 2017

Published: April 25, 2017

Copyright $\odot 2017$ by authors and Scientific Research Publishing Inc. This work is licensed under the Creative Commons Attribution International License (CC BY 4.0).

http://creativecommons.org/licenses/by/4.0/

\begin{abstract}
In the present study, we report on an efficient method for large-area photoreduction of graphene oxide flexible films. The laser-based reduction can be carried out in situ and can be tuned to attain the properties required. A systematic study has been conducted to evaluate the variation of the degree of reduction with the actual reduction temperature, which is measured using an infrared thermal camera. Local reduction temperature is varied up to $350^{\circ} \mathrm{C}$, and the degree of reduction is measured using the $\mathrm{C} / \mathrm{O}$ ratio. The $\mathrm{C} / \mathrm{O}$ ratio is increased from 2:1 for graphene oxide to 10:1 for reduced graphene oxide. This high degree of reduction is observed at low temperatures, and also in a short period of time. Thermal conductivity properties calculated using the temperature distribution shows the in-plane thermal conductivities of graphene oxide and reduced graphene oxide are a few orders of magnitude lower than single layer graphene. This can be attributed to oxygen-defect scattering, and also due to the heat conduction through the thickness of the sample by way of contact between adjacent flakes. This photoreduction method provides a way for roll-to-roll scalable production of graphene-based flexible films.
\end{abstract}

\section{Keywords}

Graphene, Graphene Oxide, Photoreduction, Laser

\section{Photoreduction of Graphene Oxide}

Graphene oxide has been demonstrated to be a good precursor material for fabrication of graphene-based materials in large quantities due to its low-cost and easily scalable synthesis process. The oxygen-containing functional groups in graphene oxide need to be removed to enable it for use in a wide range of applications. Various thermal and chemical methods are commonly used for reduction of graphene oxide. These require the use of toxic chemicals such as hydrazine, or thermal treatment at high temperatures, resulting in hazardous condi- 
tions, which are unsuitable for certain applications. To overcome the use of such hazardous means of reduction, unconventional means of heat application have been reported using a variety of light sources such as lasers and solar radiation. Photoreduction is a mild method, which is very appealing for microelectronics since a pattern of reduced graphene oxide can be formed on graphene oxide films.

Photoreduction can be achieved by two means-a photothermal process or a photochemical process. In a photothermal process, the generation of heat on exposure to high intensity light causes deoxidation of graphene oxide. In a photochemical process, a sacrificial compound is used for the reduction of graphene oxide. The UV-Vis absorption spectra show high absorption at UV and low visible wavelengths [1]. Hence, a lot of photoreduction methods developed have used lasers with wavelengths in the $200-550 \mathrm{~nm}$ regimes.

A variety of photochemical methods for the reduction of graphene oxide have been developed using UV-irradiation in the presence of various gases and catalysts. One of the first reports on photochemical reduction of graphene oxide was reported in 2011 by Kamat and group at the University of Notre Dame [2]. A suspension of graphene oxide and $\mathrm{TiO}_{2}$ nanoparticles was irradiated with a xenon lamp. They observed over an order of magnitude increase in conductivity after the reduction of graphene oxide $\mathrm{TiO}_{2}$ suspension. Another facile method was reported by Li et al. by using polyoxometalate as a catalyst [3]. This method was used by Zhang et al. for inkjet printing of multi-layer films, which were demonstrated to be excellent chemical sensors [4]. Other catalysts such as zinc oxide nanoparticles [5], silicon nanowires, and $\mathrm{BiVO}_{4}$ nanoparticles [6] have also been shown to increase the efficiency of the photochemical reduction process.

In a photothermal reduction process, the energy of the photons is converted to thermal energy, which increases the local temperature of the sample, resulting in reduction. Reduction of graphene oxide was reported by Matsumoto et al. using UV radiation in $\mathrm{H}_{2}$ or $\mathrm{N}_{2}$ at room temperature, without the use of a photocatalyst [7]. They achieved increase in conductivity by over five orders of magnitude. A group in Russia achieved photoreduction of graphene oxide films and suspensions using a mercury lamp [8] [9]. Sun's group at Jilin University in China recently reported on using a two-beam laser interference technique for patterning and reduction of graphene oxide [10] [11]. Numerous groups have reported using pulsed lasers for reduction of graphene oxide in suspension [12] [13] [14] [15] [16].

In addition to lasers as a means for photothermal reduction, novel light sources such as flash lamp and solar radiation have also been reported in the literature for graphene oxide reduction. Cote et al. reported using a xenon flash lamp to reduce graphene oxide at ambient conditions [17]. They also demonstrated patterning on graphene oxide and polymer composite films by using a photomask. Focused solar radiation, for one-pot synthesis of reduced graphene oxide and its composites, was shown to achieve conductivity values which are close to that of graphite [18]. 
Although numerous studies have been reported on using lasers for reduction of graphene oxide, a systematic study on the heat generated in the sample and the resulting rise in temperature has never been reported. The temperature distribution in the graphene oxide films and the corresponding degree of reduction can be useful in tuning the required properties of the reduced graphene oxide film obtained. In this study, the temperature distribution on the surface of the graphene oxide film when subject to a laser is captured using an infrared thermal camera. The degree of reduction, measured by the change in carbon-to-oxygen ratio, is quantified with respect to the power density and the surface temperature of the graphene oxide film. The change in conductivity is also measured using a Keithley 2400 sourcemeter.

\section{Thermal Properties of Graphene and Graphene-Based Materials}

Graphene has been shown to have superior thermal conductivity of up to 5000 $\mathrm{W} / \mathrm{m}-\mathrm{K}$, and has hence been of great interest to the scientific community to solve the severe problem of heat dissipation in microelectronics. The continuous shrinkage and increase in the number of electronic components in microelectronics has led to a thermal management problem. Due to the high thermal conductivities of carbon-based nanoscale materials such as carbon nanotubes and graphene, they have been considered as the next generation of thermal interface materials. Carbon nanotubes were used as fillers in polymeric matrices and used as thermal interface materials. However, the interface resistance at the interface between the nanotubes and the polymer limited the thermal conductivity of the composite [19] [20].

The use of graphene in polymeric composites has also been reported for thermal management [21] [22]. Although the interface resistance between graphene and polymeric matrix is considerably lower compared to nanotubes, the thermal properties are still lower than pristine graphene. This suggests that graphene and graphene-based materials can themselves be used as promising materials for thermal management. To this effect, numerous experimental, theoretical, and numerical studies have been reported in the literature for characterization of thermal properties of graphene and graphene-based materials.

Multi-layer graphene-based flexible films can be promising candidates for thermal management due to the high thermal conductivity in the direction parallel to the graphene flakes, and low thermal conductivity in the thickness direction. Thermal conductivity of graphene has been shown to decrease with increasing number of graphene layers [23] [24]. Mahanta and Abramson studied the influence of interlayer spacing and oxygen content on the thermal conductivity of graphene nanoplatelets [25]. They obtained a considerable drop from $2275 \mathrm{~W} / \mathrm{m}-\mathrm{K}$ for a sample with interlayer spacing $0.3372 \mathrm{~nm}$ to $18 \mathrm{~W} / \mathrm{m}-\mathrm{K}$ for a sample with interlayer spacing $0.3827 \mathrm{~nm}$. A significant thermal conductivity enhancement was reported by $\mathrm{Yu}$ et al. by using alkaline earth metals to modify graphene oxide papers [26]. 
Although numerous studies have been reported in the literature on the thermal transport properties of pristine graphene and graphene-based composites using numerical and experimental methods, the studies on thermal properties of graphene oxide have been very few and far-between, with considerable differences observed in the results. And since graphene oxide has been receiving a lot of attention lately as a promising precursor for graphene-based materials, it is important to characterize the thermal properties of this material. In this study, a transient heating method using a laser is used to calculate the in-plane thermal properties of graphene oxide flexible films. The temperature on the surface of the film is monitored using an infrared thermal camera.

\section{Experimental Section}

\subsection{Experimental Setup}

Graphene oxide (GO) was synthesized using the Hummers' method [27]. To prepare graphene oxide, graphite, sulphuric acid $\left(\mathrm{H}_{2} \mathrm{SO}_{4}, 98 \%\right)$, sodium nitrate $\left(\mathrm{NaNO}_{3}\right)$, potassium permanganate $\left(\mathrm{KMnO}_{4}\right)$, and hydrogen peroxide $\left(\mathrm{H}_{2} \mathrm{O}_{2}, 30\right.$ wt\%) were obtained from Alfa Aesar. Graphite, $\mathrm{NaNO}_{3}$, and $\mathrm{KMnO}_{4}$ were used as obtained. To dilute $\mathrm{H}_{2} \mathrm{SO}_{4}$ and $\mathrm{H}_{2} \mathrm{O}_{2}$ to required concentrations, Milli-Q deionized water with resistivity of $\sim 18 \mathrm{M} \Omega$-cm was used. A suspension of graphene oxide in water was prepared by ultrasonication in a bath sonicator for $2 \mathrm{~h}$. GO paper samples were prepared using bottle-top vacuum filtration of this suspension through a $47 \mathrm{~mm}$ diameter Whatman Anodisc membrane filter with a pore size of $0.02 \mu \mathrm{m}$. The GO papers were left overnight to dry at room temperature, and then peeled off from the filter. The GO films fabricated are as shown in Figure 1.

To prepare samples for photoreduction, the graphene oxide films fabricated previously were suspended between two islands, which do not conduct heat, to avoid the conduction of heat from the sample. For photoreduction, a $532 \mathrm{~nm}$ Millennia CW laser manufactured by Spectra Physics was used. The laser has a

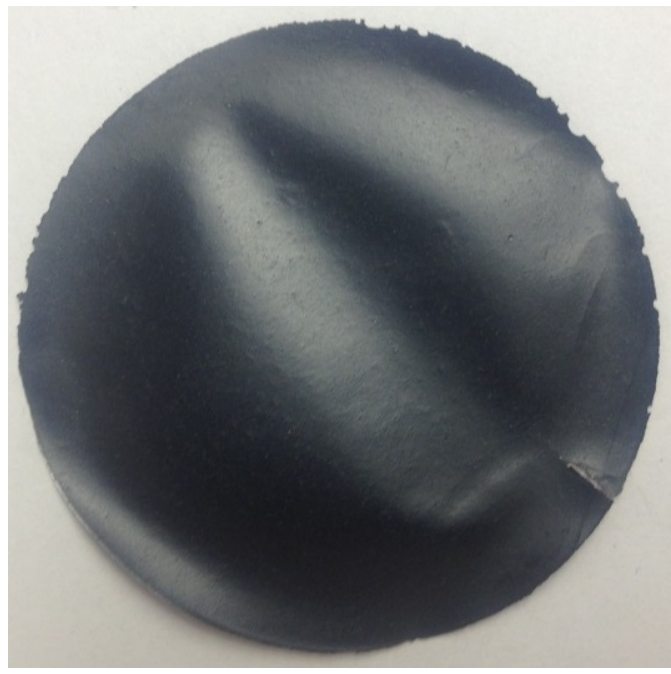

Figure 1. GO Paper. 
beam spot of $1 \mathrm{~mm}$ and a maximum power of $5 \mathrm{~W}$. To obtain a line heat source on the sample, the laser beam was passed through a beam expander and a cylindrical lens, prior to impinging on the graphene oxide film. The thermal signature was captured using a SC6000 infrared thermal camera by FLIR Systems. The camera was connected to a laptop using an ethernet cable, and data was recorded using the FLIR ExaminIR software. A schematic of the setup is shown in Figure 2.

\subsection{Experimental Procedure}

For the experiments, the laser power was varied from $65 \mathrm{~mW}$ to $400 \mathrm{~mW}$, which correspond to average power densities of $3.25 \mathrm{~W} / \mathrm{cm}^{2}$ to $20 \mathrm{~W} / \mathrm{cm}^{2}$. Data was recorded at a sampling rate of $500 \mathrm{~Hz}$. At each power level, the following procedure was used: the data recording was started, the laser was turned on for $\sim 10$ seconds, and data recording was stopped $\sim 10$ seconds after the laser is turned off to get a transient temperature response in the sample. The transient temperature data is useful to characterize the thermal properties of the sample. The reduction of the sample was characterized using energy-dispersive X-ray spectroscopy (EDS) in a Hitachi SU8030 scanning electron microscope and the electrical characterization was conducted using a 2-probe method and a Keithley 2400 sourcemeter.

\section{Results and Discussion}

\subsection{Photoreduction of Graphene Oxide Films}

Figure 3 shows a representative temperature distribution on the surface of the sample when subject to a laser power density of $3.25 \mathrm{~W} / \mathrm{cm}^{2}$. The maximum temperature, as expected, is at the center where the heat source is located. Since a line heat source is used in this case, a symmetric temperature distribution is observed with respect to the vertical. The maximum temperature on the surface of the sample was related to the incident power density. The variation of maximum temperature on the surface of the graphene oxide film with power density is shown in Figure 4. The maximum temperature on the sample surface increases linearly with a moderate slope of 11.626 till a power density of $15 \mathrm{~W} / \mathrm{cm}^{2}$.

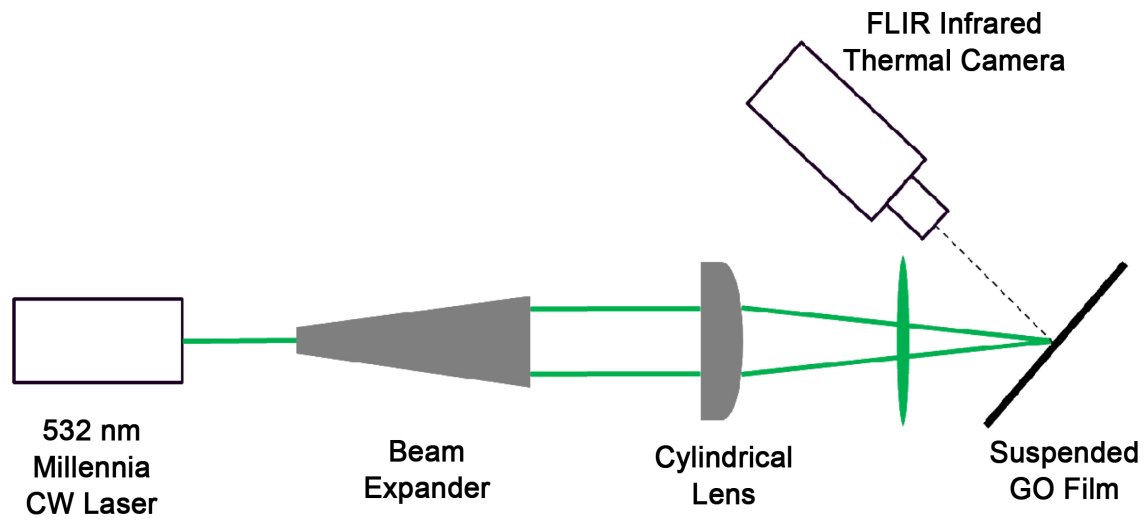

Figure 2. Experimental setup for photoreduction and temperature imaging. 


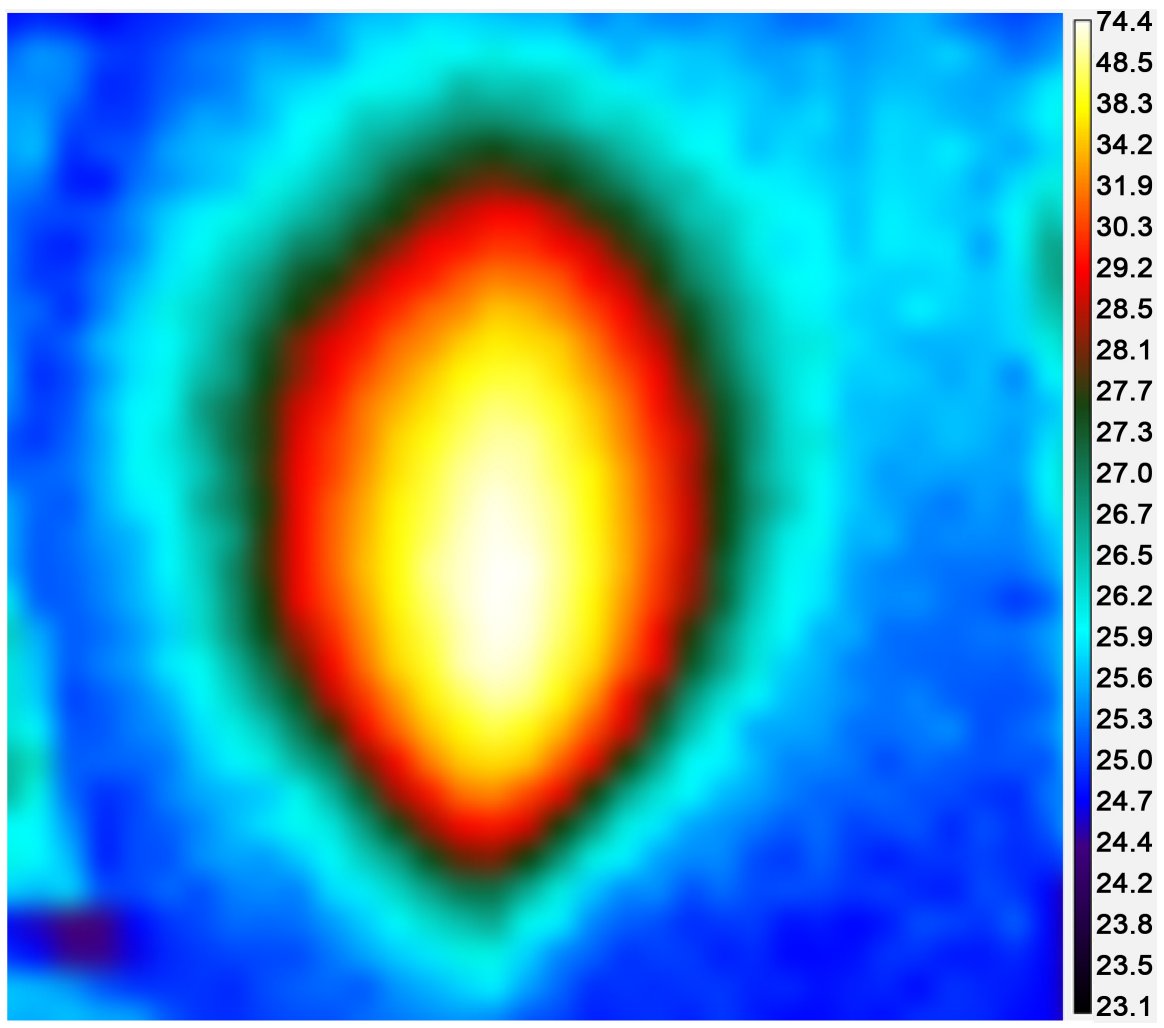

Figure 3. Representative temperature distribution on the surface of the sample (contour plot in $\left.{ }^{\circ} \mathrm{C}\right)$.

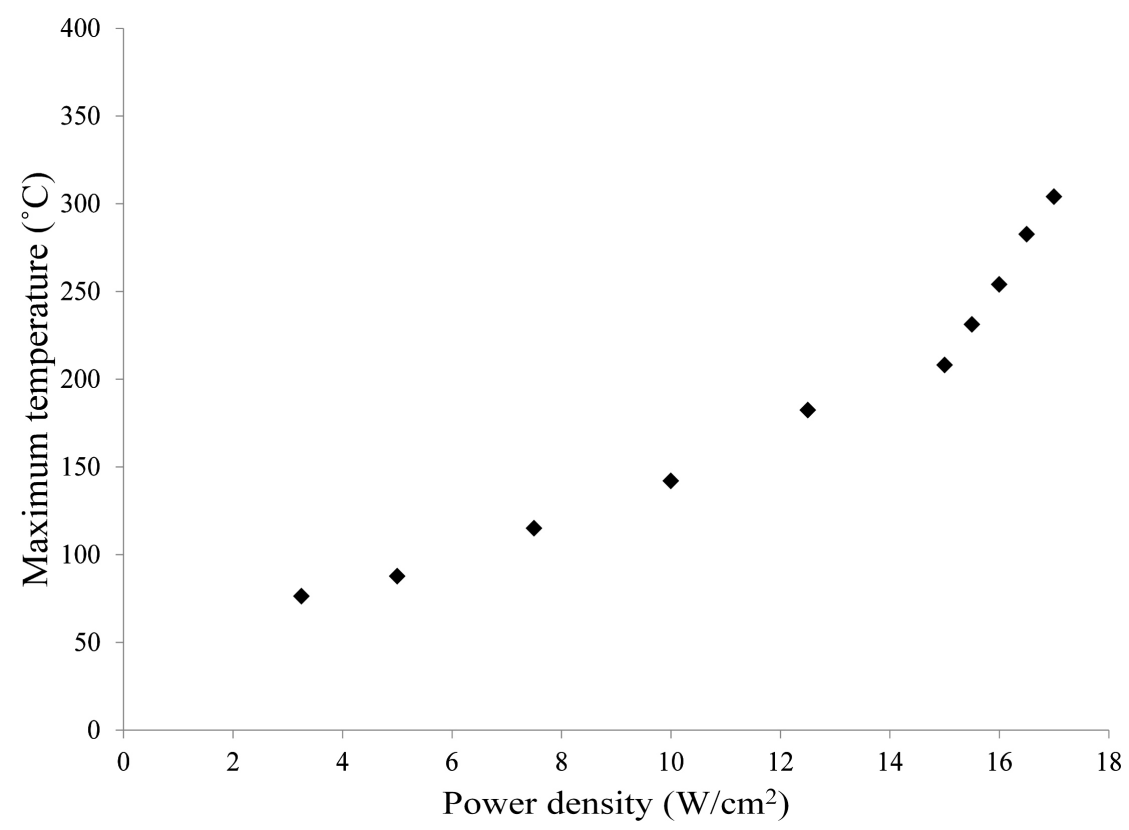

Figure 4. Variation of maximum measured surface temperature with power density.

At $15 \mathrm{~W} / \mathrm{cm}^{2}$, the slope increases to 48.7 until the power density reaches 17 $\mathrm{W} / \mathrm{cm}^{2}$. At $17.5 \mathrm{~W} / \mathrm{cm}^{2}$, the rise in temperature is sudden and exponential, which is beyond the calibrated range of the thermal camera. Trusovas et al. conducted a COMSOL simulation to show that the temperature on the surface of 
a graphene oxide sample increased to beyond $1000^{\circ} \mathrm{C}$ when the pulse energy exceeded $0.3 \mu \mathrm{J}$ [15].

Up to a power density of $17 \mathrm{~W} / \mathrm{cm}^{2}$, a controlled reduction of the graphene oxide film was observed, without exfoliation or ablation. The reduction can be quantified by measuring the change in carbon-to-oxygen ratio in the reduced sample with respect to the graphene oxide film. The carbon-to-oxygen ratio was calculated by measuring the atomic weight percentages in the reduced films using energy dispersive X-ray spectroscopy in a scanning electron microscope. Figure 5 shows a variation of the carbon-to-oxygen ratio with the surface temperature. The carbon-to-oxygen ratio increases from $\sim 2.5$ for unreduced graphene oxide to $\sim 10$ for sample heated to $340^{\circ} \mathrm{C}$, which confirms effective reduction of the sample at low temperatures.

From a combination of Figure 4 and Figure 5, it is observed that the degree of reduction increases drastically when the surface temperature of the graphene oxide film inreases beyond $\sim 200^{\circ} \mathrm{C}$. This can be due to a combination of two reasons-heating due to laser, and the heat generated due to the reduction reaction within the graphene oxide film. The reduction in the graphene oxide film is low at measured temperatures below $\sim 200^{\circ} \mathrm{C}$, and hence the surface temperature measured is only due to laser heating. At power densities above $15 \mathrm{~W} / \mathrm{cm}^{2}$, the increase in reduction of graphene oxide causes internal heat generation. The measured surface temperature is a combination of laser heating and internal heat, and hence a change in slope of the measured temperature is observed.

The conductivity of each sample was also measured using a 2-probe method with a Keithley 2400 sourcemeter. To estimate the increase in conductivity, the values were normalized to that of graphene oxide before reduction. Figure 6 shows the variation of normalized conductivity with the reduction temperature.

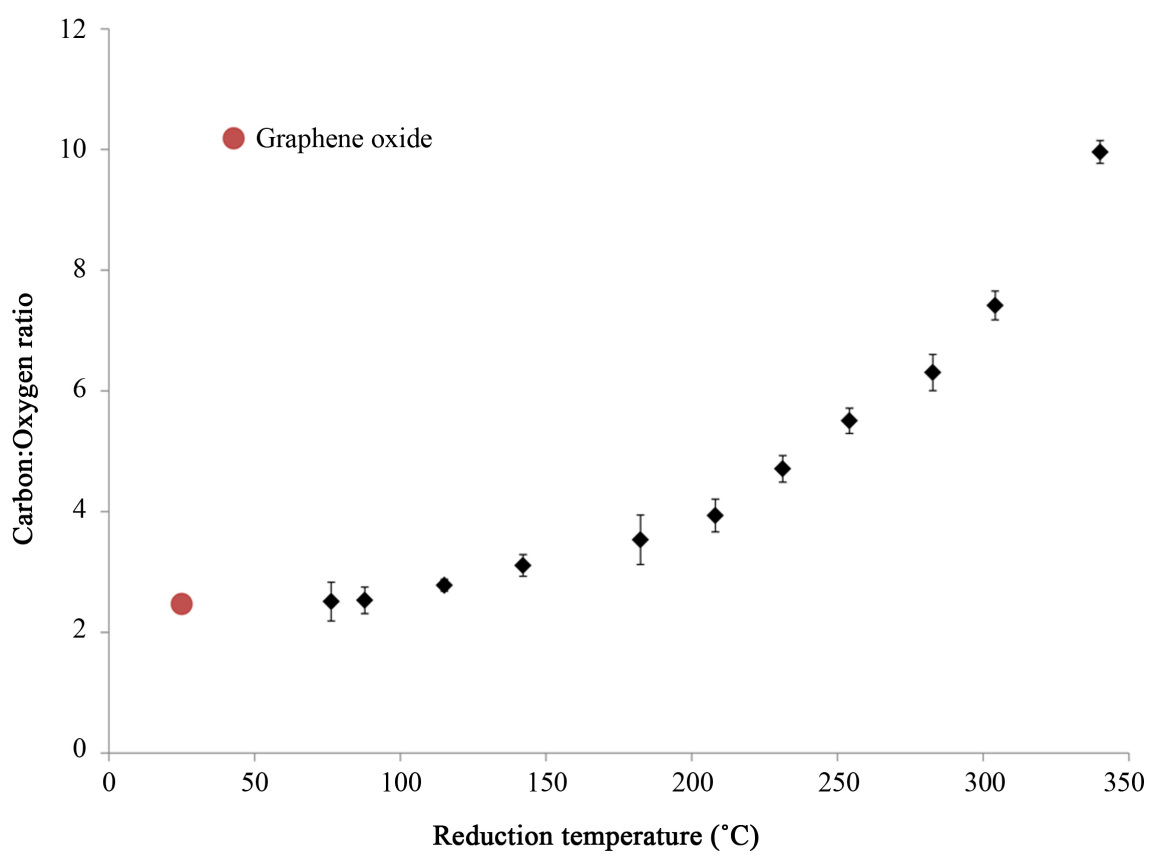

Figure 5. Variation of carbon-to-oxygen ratio with reduction temperature. 


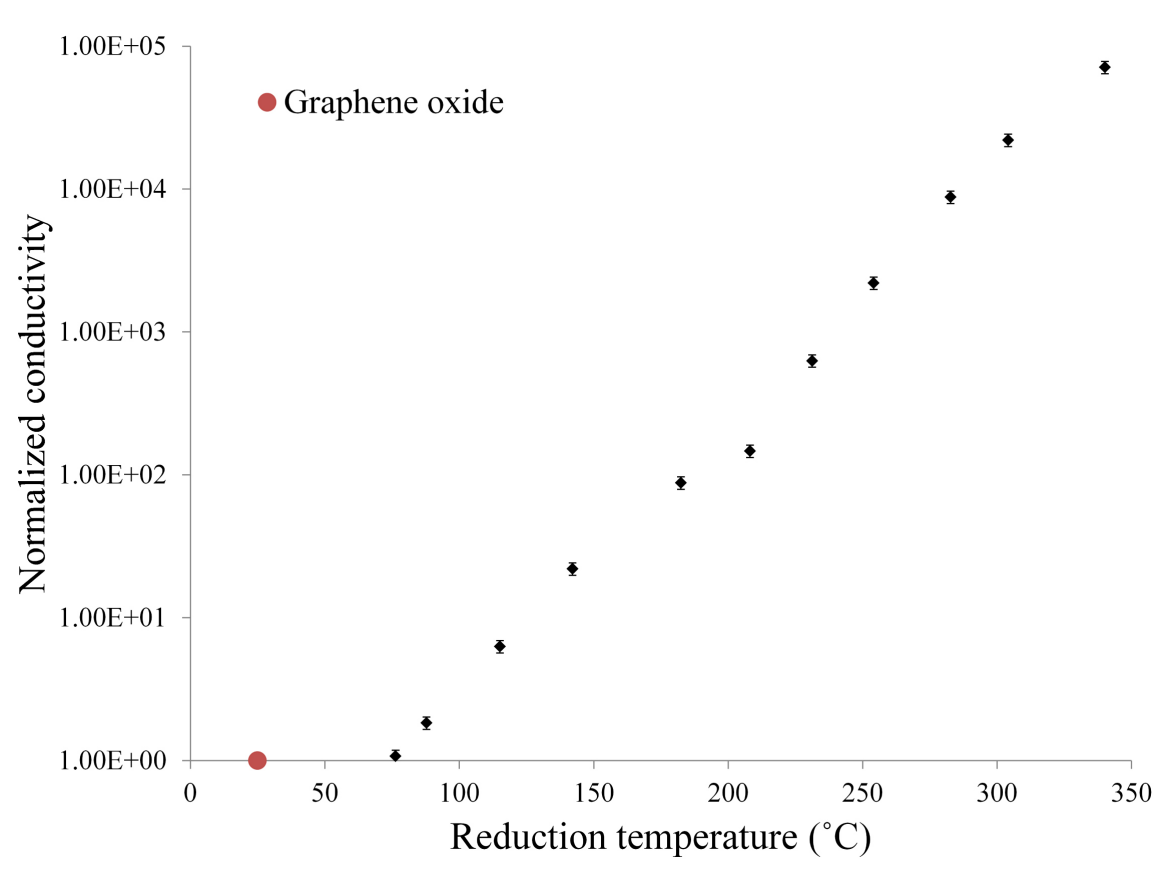

Figure 6. Variation of normalized conductivity with reduction temperature.

The conductivity increases rapidly with increasing reduction temperature. Very minimal reduction is observed below $75^{\circ} \mathrm{C}$, and hence laser power density was adjusted to a range where reduction could be observed. A change in conductivity of almost five orders of magnitude is observed for samples reduced at $\sim 350^{\circ} \mathrm{C}$.

For power densities beyond $17 \mathrm{~W} / \mathrm{cm}^{2}$, exfoliation of the sample is observed. As mentioned earlier, a COMSOL simulation shows that the local temperature increases to beyond $1000^{\circ} \mathrm{C}$, which causes a tremendous build-up of internal pressure due to the presence of oxygen-containing functional groups. Since no external pressure is used to counter the internal pressure, the film exfoliates into reduced graphene oxide flakes. The escape of gases can be seen in the sequence of images in Figure 7. A characterization of the exfoliated sample using EDS shows a carbon-to-oxygen ratio of $\sim 30$.

In a previous study, the reduction of graphene oxide was demonstrated using stress confinement [28]. The carbon-to-oxygen ratio obtained in this study is higher than that obtained by thermal reduction using stress confinement at the same temperature. This might be attributed to two reasons-the actual temperature on the surface of the sample, and the role of platens used for stress confinement. The infrared thermal camera captures the actual temperature on the surface of the sample, and hence the exact temperature to which the sample is being subjected. Normally, thermal treatment is conducted in a tube furnace, or as in the case mentioned in the reference article, in an atmospheric furnace. The temperature is measured by thermocouples situated at various points inside the furnace. There is a possibility for the temperature to vary inside the furnace, and the actual temperature that the sample is subjected to might be lower than the recorded furnace temperature. Hence, although the literature suggests a high furnace temperature is required for reduction of graphene oxide, the actual surface 

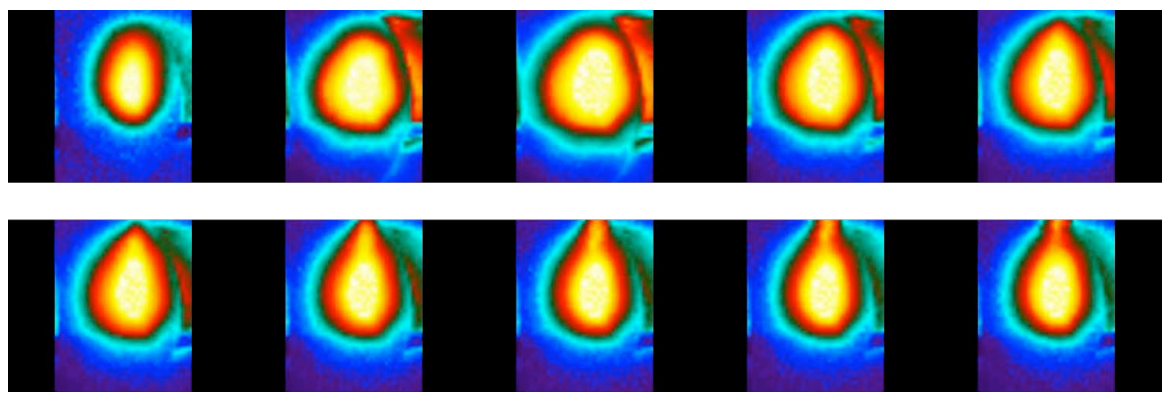

Figure 7. Exfoliation of film on exposure to high power densities.

temperature required for effective reduction is lower, as is seen from Figure 8.

Using laser-based photoreduction, it has been observed that at power densities less than $17 \mathrm{~W} / \mathrm{cm}^{2}$, controlled and effective reduction of graphene oxide films can be achieved without exfoliation into small flakes. The use of compression platens reduces the available pathways for oxygen functional groups to escape the graphene oxide film. In this laser-based reduction technique, since no stress confinement is used, the oxygen-based functional groups are free to escape through the thickness of the film, as well as laterally. Since more oxygen leaves the film due to the higher number of pathways, a higher carbon-to-oxygen ratio is observed at a similar temperature.

There are various advantages to using this method for reduction of graphene oxide films. This method of reduction can be used to tune the degree of reduction required to obtain the required properties. Since a line heat source is used, a larger area can be reduced, which is suitable for roll-to-roll and scalable fabrication of reduced graphene oxide films. It can also be used to reduce only a part of the film, while keeping other parts unreduced. Also, the reduction is achieved in a few seconds, making it a fast, energy efficient, and safe process.

\subsection{Thermal Properties of Graphene Oxide Films}

The thermal properties of graphene oxide films can be calculated by using the variation of temperature at various points on the film against time. Figure 8 shows representative normalized curves of the temperature variation with time, which are extracted from the data captured by the thermal camera. The curves show a fast rise of temperature after the laser is turned on, and a similar decay after the laser is switched off.

The line-source transient heat conduction method is one of the most widely used to calculate the thermal conductivities of materials. For small distances away from the heating source, the variation of temperature with time is given by Equation (1), where $A$ is a constant, $Q$ is the heat input per unit length $(\mathrm{W} / \mathrm{m})$, and $k$ is the thermal conductivity $(\mathrm{W} / \mathrm{m}-\mathrm{K})$ [29]. The slope of a plot of temperature versus the natural logarithm of time can be used to calculate the in-plane thermal conductivity of the film. Using this method, an average in-plane thermal conductivity of $2.23 \mathrm{~W} / \mathrm{m}-\mathrm{K}$ is obtained for the film, which is a few orders of magnitude lower than single layer graphene, but close to that reported by $\mathrm{Yu}$ et al. for graphene oxide [26]. 


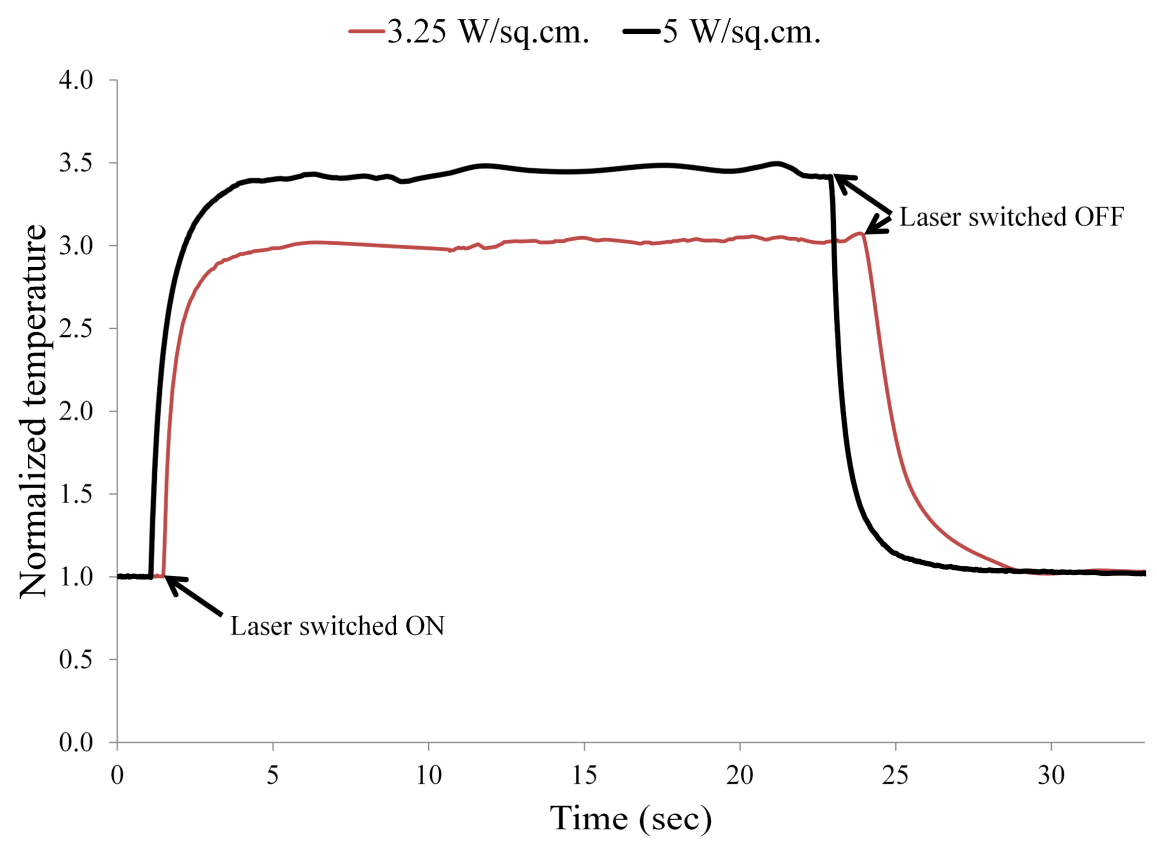

Figure 8. Representative normalized temperature versus time curves.

$$
T=A+\frac{Q}{4 \pi k} \ln (t)
$$

The above method requires the use of the average heat input per unit length, which cannot be accurately calculated using the current setup. Parker et al. described another method for determining the thermal diffusivity, heat capacity, and thermal conductivity of materials, which does not require the knowledge of the heat input [30]. The thermal diffusivity can be calculated using Equation (2), where $\alpha$ is the thermal diffusivity $\left(\mathrm{m}^{2} / \mathrm{s}\right), L$ is the distance from the heat source $(\mathrm{m})$, and $t_{1 / 2}$ is the time required for the point to reach half the maximum temperature (s). Thermal conductivity is then calculated by the product of the thermal diffusivity, specific heat capacity, and density (Equation (3)). The specific heat capacity and density are assumed to be $710 \mathrm{~J} / \mathrm{kg}-\mathrm{K}$ and $1800 \mathrm{~kg} / \mathrm{m}^{3}$ respectively [25] [31]. This gives the average thermal conductivity of graphene oxide films as $1.89 \mathrm{~W} / \mathrm{m}-\mathrm{K}$.

$$
\begin{aligned}
& \alpha=\frac{1.38 L^{2}}{\pi^{2} t_{1 / 2}} \\
& k=\alpha \cdot C_{p} \cdot \rho
\end{aligned}
$$

The thermal conductivities for layered graphene oxide films are considerably lower than those for single layer graphene flakes. This can be due to the availability of more phonon modes for scattering, as well as the conductivity through the thickness of the sample. However, the values obtained in this study are close to those reported in the literature. Yu et al. reported thermal conductivity values of $3.91 \mathrm{~W} / \mathrm{m}-\mathrm{K}$ for graphene oxide using a nanoflash technique [26]. Mu et al. used molecular dynamics simulations, and reported a thermal conductivity of 8.8 $\mathrm{W} / \mathrm{m}-\mathrm{K}$ for graphene oxide with oxygen coverage of $20 \%$ [32]. They proposed that 
oxygen defect scattering is the dominating reason for the low thermal conductivity of graphene oxide. In this study, the oxygen coverage is higher than $30 \%$, which might explain the lower thermal conductivities obtained.

The thermal conductivities of reduced graphene oxide films were calculated using a similar method. The films were first reduced at different temperatures, and were then subjected to a laser flash at low power density. The low power density prevents further reduction of the sample, but does give the transient temperature response data, which is used to calculate the thermal conductivity. As seen from Figure 9, the thermal conductivity increases exponentially from $\sim 2$ $\mathrm{W} / \mathrm{m}-\mathrm{K}$ for graphene oxide, to $\sim 40 \mathrm{~W} / \mathrm{m}-\mathrm{K}$ for graphene oxide reduced at $340^{\circ} \mathrm{C}$. This is still a couple of orders of magnitude lower than pristine graphene, suggesting that scattering and through thickness transport are still the dominant mechanisms for thermal transport in multi-layered graphene films.

\section{Summary}

An efficient method for large-area photoreduction of graphene oxide flexible films is reported in this article. The reduction can be carried out in situ and can be tuned to attain the properties required. For the first time, a systematic study has been conducted to evaluate the variation of the degree of reduction with the actual reduction temperature, which is measured using an infrared thermal camera. A high degree of reduction is observed at low temperatures, and also in a short period of time as opposed to previous studies reported in the literature. The temperature distribution data obtained using the thermal camera is also used to calculate the thermal conductivity of graphene oxide films. In-plane thermal conductivities of graphene oxide and reduced graphene oxide are a few orders of magnitude lower than single layer graphene. This can be attributed to

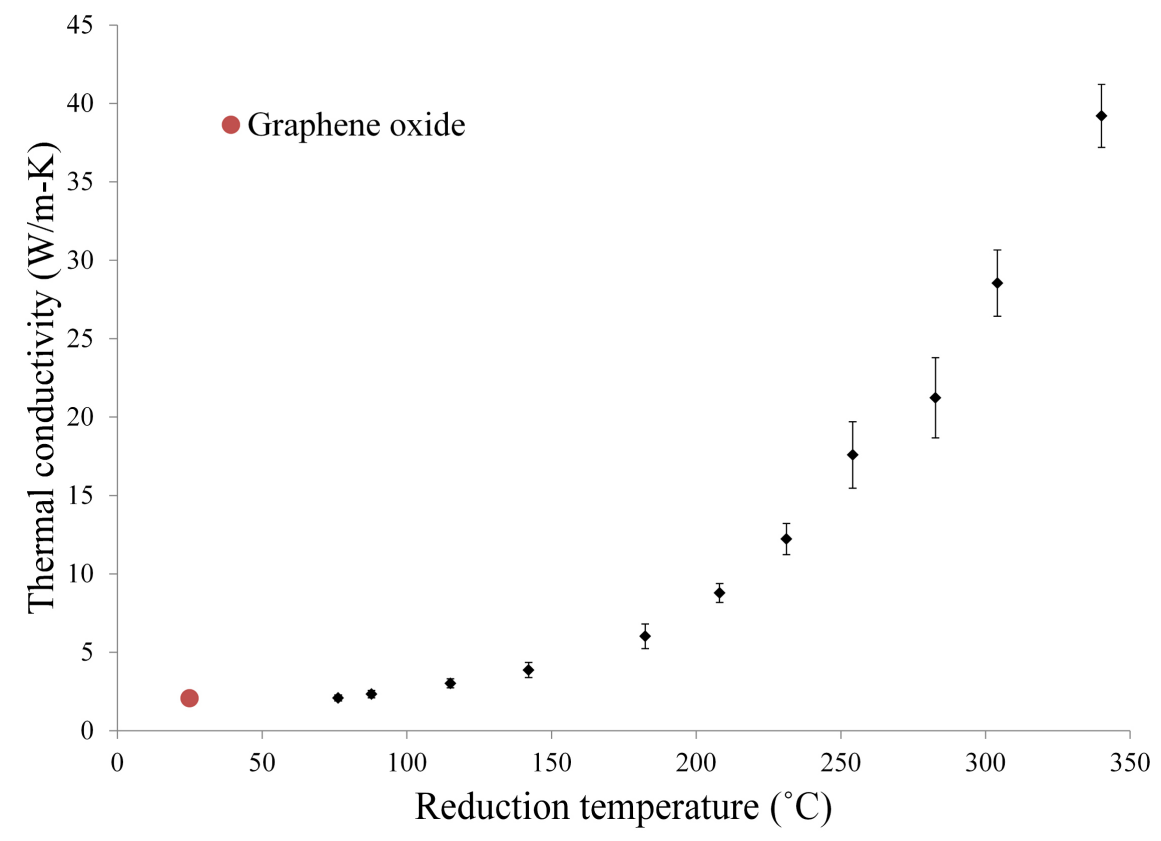

Figure 9. Thermal conductivity of reduced graphene oxide films. 
oxygen-defect scattering, and also due to the heat conduction through the thickness of the sample by way of contact between adjacent flakes. This photoreduction method provides a way for roll-to-roll scalable production of graphene-based flexible films.

\section{Acknowledgements}

This work was supported in part by the National Science Foundation through grant OISE-0730259, and in part by the Department of Transportation through the Infrastructure Technology Institute at Northwestern University. This work made use of Central Facilities supported by the MRSEC program of the National Science Foundation at the Northwestern University Materials Research Science and Engineering Center. The SEM and EDX work was performed in the EPIC facility of the NUANCE Center at Northwestern University. NUANCE Center is supported by NSF-NSEC, NSF-MRSEC, Keck Foundation, the State of Illinois, and Northwestern University.

\section{References}

[1] Li, D., Müller, M.B., Gilje, S., Kaner, R.B. and Wallace, G.G. (2008) Processable Aqueous Dispersions of Graphene Nanosheets. Nature Nanotechnology, 3, 101-105. https://doi.org/10.1038/nnano.2007.451

[2] Williams, G., Seger, B. and Kamat, P.V. (2008) $\mathrm{TiO}_{2}$-Graphene Nanocomposites. UV-Assisted Photocatalytic Reduction of Graphene Oxide. ACS Nano, 2, 14871491. https://doi.org/10.1021/nn800251f

[3] Li, H.L., Pang, S.P., Feng, X.L., Müllen, K. and Bubeck, C. (2010) Polyoxometalate Assisted Photoreduction of Graphene Oxide and Its Nanocomposite Formation. Chemical Communications, 46, 6243-6245. https://doi.org/10.1039/c0cc01098g

[4] Zhang, H., Xie, A.J., Shen, Y.H., Qiu, L.G. and Tian, X.Y. (2012) Layer-by-Layer Inkjet Printing of Fabricating Reduced Graphene-Polyoxometalate Composite Film for Chemical Sensors. Physical Chemistry Chemical Physics, 14, 12757-12763. https://doi.org/10.1039/c2cp41561e

[5] Williams, G. and Kamat, P.V. (2009) Graphene-Semiconductor Nanocomposites: Excited-State Interactions between $\mathrm{ZnO}$ Nanoparticles and Graphene Oxide. Langmuir, 25, 13869-13873. https://doi.org/10.1021/la900905h

[6] Ng, Y.H., Iwase, A., Kudo, A. and Amal, R. (2010) Reducing Graphene Oxide on a Visible-Light $\mathrm{BiVO}_{4}$ Photocatalyst for an Enhanced Photoelectrochemical Water Splitting. Journal of Physical Chemistry Letters 1, 2607-2612. https://doi.org/10.1021/jz100978u

[7] Yasumichi Matsumoto et al. (2010) Simple Photoreduction of Graphene Oxide Nanosheet under Mild Conditions. ACS Applied Materials \& Interfaces, 2, 3461- 3466. https://doi.org/10.1021/am100900q

[8] Smirnov, V.A., et al. (2011) Photoreduction of Graphite Oxide. High Energy Chemistry, 45, 57-61. https://doi.org/10.1134/S0018143911010176

[9] Plotnikov, V.G., Smirnov, V.A., Alfimov, M.V. and Shulga, Y.M. (2011) The Graphite Oxide Photoreduction Mechanism. High Energy Chemistry, 45, 411-415. https://doi.org/10.1134/S0018143911050158

[10] Guo, L, et al. (2012) Two-Beam-Laser Interference Mediated Reduction, Patterning and Nanostructuring of Graphene Oxide for the Production of a Flexible Humidity 
Sensing Device. Carbon, 50, 1667-1673,

[11] Wang, J.-N., et al. (2012) Biomimetic Graphene Surfaces with Superhydrophobicity and Iridescence. Chemistry-An Asian Journal, 7, 301-304.

https://doi.org/10.1002/asia.201100882

[12] Chang, H.-W., Tsai, Y.-C., Cheng, C.-W., Lin, C.-Y. and Wu, P.-H. (2012) Reduction of Graphene Oxide in Aqueous Solution by Femtosecond Laser and Its Effect on Electroanalysis. Electrochemistry Communications, 23, 37-40.

[13] Ghadim, E.E., et al. (2014) Pulsed Laser Irradiation for Environment Friendly Reduction of Graphene Oxide Suspensions. Applied Surface Science, 301, 183-188.

[14] Petridis, C., et al. (2013) Post-Fabrication, in Situ Laser Reduction of Graphene Oxide Devices. Applied Physics Letters, 102, Article ID: 093115. https://doi.org/10.1063/1.4794901

[15] Trusovas, R., et al. (2013) Reduction of Graphite Oxide to Graphene with Laser Irradiation. Carbon, 52, 574-582.

[16] Zhang, Y., et al. (2010) Direct Imprinting of Microcircuits on Graphene Oxides Film by Femtosecond Laser Reduction. Nano Today, 5, 15-20.

[17] Cote, L.J., Cruz-Silva, R. and Huang, J. (2009) Flash Reduction and Patterning of Graphite Oxide and Its Polymer Composite. Journal of the American Chemical Society, 131, 11027-11032. https://doi.org/10.1021/ja902348k

[18] Eswaraiah, V., Sasikaladevi, S., Aravind, J. and Ramaprabhu, S. (2011) Top down Method for Synthesis of Highly Conducting Graphene by Exfoliation of Graphite Oxide Using Focused Solar Radiation. Journal of Materials Chemistry, 21, 68006803. https://doi.org/10.1039/c1jm10808e

[19] Nan, C.-W., Shi, Z. and Lin, Y. (2003) A Simple Model for Thermal Conductivity of Carbon Nanotube-Based Composites. Chemical Physics Letters, 375, 666-669.

[20] Deng, F., Zheng, Q.-S., Wang, L.-F. and Nan, C.-W. (2007) Effects of Anisotropy, Aspect Ratio, and Nonstraightness of Carbon Nanotubes on Thermal Conductivity of Carbon Nanotube Composites. Applied Physics Letters, 90, Article ID: 021914. https://doi.org/10.1063/1.2430914

[21] Yu, A., Ramesh, P., Itkis, M.E., Bekyarova, E. and Haddon, R.C. (2007) Graphite Nanoplatelet-Epoxy Composite Thermal Interface Materials. The Journal of Physical Chemistry C, 111, 7565-7569. https://doi.org/10.1021/jp071761s

[22] Shahil, K.M.F. and Balandin, A.A. (2012) Graphene-Multilayer Graphene Nanocomposites as Highly Efficient Thermal Interface Materials. Nano Letters, 12, 861 867. https://doi.org/10.1021/nl203906r

[23] Ghosh, S., et al. (2010) Dimensional Crossover of Thermal Transport in Few-Layer Graphene. Nature Materials, 9, 555-558. https://doi.org/10.1038/nmat2753

[24] Zhong, W.-R., Zhang, M.-P., Ai, B.-Q. and Zheng, D.-Q. (2011) Chirality and Thickness-Dependent Thermal Conductivity of Few-Layer Graphene: A Molecular Dynamics Study. Applied Physics Letters, 98, Article ID: 113107. https://doi.org/10.1063/1.3567415

[25] Mahanta, N.K. and Abramson, A.R. (2012) Thermal Conductivity of Graphene and Graphene Oxide Nanoplatelets. 13 th IEEE Intersociety Conference on Thermal and Thermomechanical Phenomena in Electronic Systems, San Diego, 30 May-1 June 2012, 1-6. https://doi.org/10.1109/ITHERM.2012.6231405

[26] Yu, W., Xie, H., Li, F., Zhao, J. and Zhang, Z. (2013) Significant Thermal Conductivity Enhancement in Graphene Oxide Papers Modified with Alkaline Earth Metal Ions. Applied Physics Letters, 103, Article ID: 141913.

https://doi.org/10.1063/1.4824346 
[27] Hummers, W.S. and Offeman, R.E. (1958) Preparation of Graphitic Oxide. Journal of the American Chemical Society, 80, 1339-1339. https://doi.org/10.1021/ja01539a017

[28] Naik, G., Kaniyoor, A., Ramaprabhu, S. and Krishnaswamy, S. (2013) Large-Area Graphene-Based Thin Films Using Rapid Reduction of Graphene-Oxide. Sensors and Smart Structures Technologies for Civil, Mechanical, and Aerospace Systems, San Diego, 10-14 March 2013, 86921C. https://doi.org/10.1117/12.2012198

[29] Yang, W., Sokhansanj, S., Tang, J. and Winter, P. (2002) Determination of Thermal Conductivity, Specific Heat and Thermal Diffusivity of Borage Seeds. Biosystems Engineering, 82, 169-176.

[30] Parker, W.J., Jenkins, R.J., Butler, C.P. and Abbott, G. L. (1960) Flash Method of Determining Thermal Diffusivity, Heat Capacity, and Thermal Conductivity. Journal of Applied Physics, 32, 1679-1684. https://doi.org/10.1063/1.1728417

[31] Dikin, D.A., et al. (2007) Preparation and Characterization of Graphene Oxide Paper. Nature, 448, 457-460. https://doi.org/10.1038/nature06016

[32] Mu, X., Wu, X., Zhang, T., Go, D.B. and Luo, T. (2014) Thermal Transport in Graphene Oxide-From Ballistic Extreme to Amorphous Limit. Scientific Reports, 4, 3909. https://doi.org/10.1038/srep03909

Submit or recommend next manuscript to SCIRP and we will provide best service for you:

Accepting pre-submission inquiries through Email, Facebook, LinkedIn, Twitter, etc. A wide selection of journals (inclusive of 9 subjects, more than 200 journals)

Providing 24-hour high-quality service

User-friendly online submission system

Fair and swift peer-review system

Efficient typesetting and proofreading procedure

Display of the result of downloads and visits, as well as the number of cited articles

Maximum dissemination of your research work

Submit your manuscript at: http://papersubmission.scirp.org/

Or contact graphene@scirp.org 